\title{
Myricetin and its derivative M10, myricetin-3-0- $\beta$-d-lactose sodium salt, modify the composition of gut microbiota in mice with chronic ulcerative colitis
}

\author{
Rong-Rong Miao \\ Capital Medical University \\ Sheng Zhan \\ Capital Medical University \\ Xue-Tao Hu \\ Capital University of Medical Sciences: Capital Medical University \\ Xiao-Ling Zhou \\ Capital Medical University \\ Shu-Xiang Cui \\ Capital Medical University \\ Xian-Jun Qu ( $\nabla$ qxj@ccmu.edu.cn ) \\ Capital Medical University https://orcid.org/0000-0001-5943-3490
}

\section{Research}

Keywords: Myricetin, Derivative M10, Gut microbiota, Ulcerative colitis, Probiotics, Pathogens

Posted Date: October 2nd, 2020

DOI: https://doi.org/10.21203/rs.3.rs-84103/v1

License: @ (i) This work is licensed under a Creative Commons Attribution 4.0 International License. Read Full License 


\section{Abstract}

Background Previous studies revealed that Myricetin and derivative M10, Myricetin-3-0- $\beta$-d-lactose sodium salt, prevented chronic ulcerative colitis (UC) in mice. We investigated whether the inhibitory effects of Myricetin and M10 on UC were associated to the modification of intestinal microbiota. Samples of intestinal microbiota were collected from the ileocecum of UC mice which demonstrated response to the treatment of Myricetin and M10. Gut microbiota was analyzed by $16 \mathrm{~S}$ rDNA sequencing assay. Results UC model mice demonstrated the increases of Firmicutes and Actinobacteria as compared to healthy control mice. Oral M10 and Myricetin normalized the composition of Firmicutes and Actinobacteria. At genus level, the effect of M10 and Myricetin on ulcerative colitis was strongly associated to the increase of probiotics, such as Akkermansia, and the inhibition of pathogenic microorganisms, such as Ruminococcus and Parabacteroides. Myricetin's derivative M10 significantly increased both biosynthesis and degradation activities, resulting to strong improvements of the metabolism of sulfur, pyruvate, steroid biosynthesis and unsaturated fatty acid biosynthesis in gut microenvironment. Conclusions Natural product Myricetin and its derivative M10 could modify the modification of gut microbiota in UC mice. Combined with pharmacologic effects of Myricetin and M10 in these UC mice, we conclued that the effects of Myricetin and M10 on UC were associated to the modification of intestinal microbiota in the environment of chronic ulcerative colitis.

\section{Background}

Inflammatory bowel diseases (IBD) describe the diseases of chronic inflammation and ulceration of mucosal and submucosal layers [1, 2]. Chronic ulcerative colitis (UC), the most common forms of IBD, manifests clinically as contiguous, superficial mucosal inflammation in colon, originating at the rectum and moving toward the proximal colon [3]. Although the etiology of UC remains unknown, several factors including immune dysfunction [4, 5], environmental exposures and more recently, compositional changes of gut microbiota (i.e. dysbiosis) [6-9] have been considered to associate with the development of disease. The dysbiosis of intestinal microbiota in UC patients was described in terms of richness and diversity [10]. Richness delineates the unique number of bacteria present in a microbial community and diversity extends this to account for their relative abundance. In UC patients, the richness and the diversity were reported the reduction as compared to healthy people. These changes of gut microbiota composition indicated the prognostic value, since the declines in both metrics were associated with an increased risk of disease relapse. Therefore, to modify gut microbiota in the patients with dysbiosis have been associated with therapeutic outcomes in the patients with UC and other IBD [11-13].

Myricetin, the extensively studied polyphenol, presents abundantly in various fruits and vegetables. Numerous studies showed that Myricetin exhibits diverse pharmacological properties including anti-chronic inflammation, anticarcinogenesis and angiogenesis, and even prevention of cancers [14-16]. Recently, Myricetin was found to have the biological activity of modulation gut microbiota in rats [17]. However, Myricetin does not meet the requirements as a therapeutic drug because of substantial limitations, such as complex isolation procedures, poor water solubility $(<$ $100 \mathrm{ng} / \mathrm{ml}$ ) and low bioavailability [18]. Previously, a series of novel compounds of Myricetin were designed in our group [19]. M10 is Myricetin-3-O-b-D-lactose sodium salt by adding a hydrophilic glycosylation group. M10 was found to have a higher efficacy of anti-UC and UC-induced colorectal tumors than Myricetin [20]. M10 inhibited chronic UC through inhibiting the IL-6 and NF-KB pathways in the mucosa of colorectum [21]. More evidences are needed to demonstrate the biological activity of Myricetin and its derivative M10 in the prevention of chronic UC.

We have noted that changes of gut microbiota play important roles in the development of UC. UC patients were found significant reduction of bacterial abundance and diversity in gut microbiota, which can be described as a general pattern for fewer Firmicutes, particularly Clostridial cluster XIVa and IV, and Bacteroidetes, while more Proteobacteria [22, 23]. In DSS-induced ulcerative colitis mice, the abundance of bacteria from Firmicutes phylum was significantly decreased, while Bacteroides was consequently increased [24]. In our study, Myricetin and M10 by orally demonstrated strong acitvities of prevention UC in mice model [19]. We further analyzed the composition of gut microbiota in the ileocecum of these mice.

Page 2/14 
Both Myricetin and M10 reduced the proportions of Ruminococcue, Olsenella, Parvibacter. The composition of Akkermansia in the M10-treated mice were more abundant than control mice, and the proportions of Desulfovibrio, Parasutterella, Acetatifactor, Oscillibacter, Barnesiella, Parabacteroides, Flavonifractor, Alistipes, Lactobacillus, Prevotella, Odoribacter and Saccharibacteria_genera_incertae_sedis were prevented. Moreover, M10 increased both biosynthesis and degradation activities to improve gut microenvironment, including sulfur metabolism, pyruvate metabolism, steroid biosynthesis and unsaturated fatty acid biosynthesis. These results provided more evidences of Myricetin and M10 in the prevention of UC through modifying gut microbiota by increasing probiotics and inhibiting pathogens.

\section{Methods}

\section{Chemicals}

Myricetin (purity $>99 \%$ ) was purchased from Sigma Chemical Co. M10 (purity $\geq 99 \%$ ) was provided by Dr. Wang in Marine Biomedical Research Institute of Qingdao. Chemical structures of Myricetin and M10 were issued in the references [19, 20]. Mesalazine was purchased from Anhui Dongsheng Pharmaceutical Co. Ltd. Both drugs were dissolved in 0.5\% sodium carboxymethyl cellulose (CMC-Na) (Solarbio, China).

\section{Mice model of UC and drug treatment}

Male C57BL/ 6 mice of 6-8 weeks were purchased from Charles River Laboratories (Beijing, China). Mice were caged under controlled room temperature, humidity and light (12/12h light/dark cycle) and allowed unrestricted access to standard mouse chow and tap water [25].

A total of 70 C57BL/ 6 mice were adapted to facility for one week, and then were randomly divided into following groups (n = 10 for each group): Group 1 (healthy control group, NEG group): normal control mice (distilled water); Group 2 (UC model group, M group) : DSS-induced colitis model mice (distilled water); Group 3 (Mesalazine-treated group, POS group) : mice exposed to DSS treated with $100 \mathrm{mg} / \mathrm{kg}$ of Myricetin; Group 4-6: mice exposed to DSS treated with 25, 50 (M10-treated group, MID group) and $100 \mathrm{mg} / \mathrm{kg}$ of M10, respectively; Group 7 (Myricetin-treated group, DMY group) : mice exposed to DSS treated with control drug Mesalazine $(100 \mathrm{mg} / \mathrm{kg})$. Mice were received water containing 1.0\% DSS (36-50 kD, MP Biomedicals, LLC, Santa Ana, CA) for 7 days, and then given regular water for 14 days, followed by three additional DSS cycles [20]. Mice received either vehicle (CMC-Na) or drugs by gavage three days after DSS. Administrations were performed six times per week for 12 consecutive weeks.

\section{Sample collection and DNA extraction}

Stool samples were collected from the ileocecum of mice in standard $1.5 \mathrm{ml}$ sterile tubes and frozen immediately in liquid nitrogen. Eight samples of each group were randomly thawed and DNA was extracted using QIAamp Fast DNA Stool Mini Kit (QIAGEN, Cat.No.51604), according to the manufacturer's instructions. DNA concentration and purity were monitored on $1 \%$ agarose gel.

\section{S rDNA sequencing assay}

Amplification of variable region V3-V4 from 16S rDNA gene was performed using specific primer with barcode (Forward primer (5'-3'): CCTACGGGRSGCAGCAG (341F); Reverse prime (5'-3'): GGACTACVVGGGTATCTAATC (806R)). All PCR reactions were performed with 2X KAPA HiFi ${ }^{\mathrm{TM}}$ HotStart ReadyMix [26]. After $2 \%$ agarose gel electrophoresis, the amplified products were recycled with Axy Prep DNA gel extraction kit. Library was qualified by Thermo NanoDrop 2000 Spectrophotometer UV trace and $2 \%$ agarose gel electrophoresis and quantified by Qubit ${ }^{\circ}$ dsDNA HS assay kit. According to the amount of sequencing required for each sample, mixing ratio corresponding to predicted sequence libraries. The enriched library was sequenced on an Illumina NovaSeq PE250 platform according to manufacturer's specifications to 
generate paired-end reads around 200-420bp for further analysis. Number of reads per sample ranged from 33112 to 38452 , with an average of 35578 reads per sample.

\section{Bioinformatic analysis of 16S rRNA sequencing data}

Bioinformatic analysis of bacterial 16S rRNA amplicon data was conducted using a combination of QIIME software and the usearch platform. Paired-end raw sequences were qualified to get clean reads [27]. After filtration and chimera checking, reads were clustered into operational taxonomic units (OTUs) at 97\% identity [28]. Representative sequences for each OTU was screened for further annotation. Species-richness and alpha diversity were analysed by QIIME software [29]. Principal Coordinates Analysis (PCoA) ordination plot on weighted UniFrac distance matrix was used to describe Beta diversity difference. As for LEfSe analysis, linear discriminant analysis (LDA) scores were used to describe the difference among five groups of mice [30].

\section{Statistical analysis}

Statistical analysis was performed using GraphPad Prism 8.0.2 (GraphPad Software, San Diego, CA, USA). All values are expressed as mean \pm standard deviation (SD). After checking data for normal distribution and variance homogeneity, continuous data were compared using Mann-Whitney $U$ test, multiple Student t tests or One-way ANOVA. All $p$ values are two-tailed, and $p$ values $<0.05$ were considered significant $\left(* \mathrm{P}<0.05,{ }^{*} \mathrm{P}<0.01,{ }^{*} * \mathrm{P}<0.001\right.$ and $\left.{ }^{* \star * * P}<0.0001\right)$.

\section{Results}

\section{Myricetin and M10 reshaped gut microbiome taxonomic diversity in mice}

All pharmacological effects of Myricetin and M10 in mice were confirmed and issued in these UC mice [19]. To characterize the composition of gut microbiome in these UC mice, we randomly selected and analyzed eight stool samples of each group. Each stool sample was subjected to 16S rDNA Amplicon sequencing, which was followed by profiling of microbial community taxonomic composition and potential function. 16S rDNA Amplicon sequencing retrieved an overall number of 1423136 clean reads, which were clustered in 10360 operational taxonomic units (OTUs) according to strict criteria. Goods coverage of all samples was greater than $99.9 \%$ (Table 1), indicating that this sequencing reflects the true nature of intestinal flora. Over $99 \%$ of sampled sequences belonged to 6 phyla: Firmicutes $(38.80 \pm 7.83 \%)$, Verrucomicrobia (31.20 \pm 18.84\%), Bacteroidetes (23.19 $\pm 15.25 \%)$, Proteobacteria $(4.07 \pm 3.71 \%)$, Actinobacteria ( $2.49 \pm$ $0.83 \%)$, and Candidatus Saccharibacteria $(0.21 \pm 0.11 \%)$ (Fig. $1 \mathrm{~A})$.

Table 1

The index of richness and diversity of gut microbiota in every group

\begin{tabular}{|llllll|}
\hline Alpha diversity & Healthy control & UC model & Mesalazine & M10 & Myricetin \\
\hline Chao1 & $312.76 \pm 14.67$ & $284.99 \pm 11.46$ & $283.61 \pm 27.87$ & $257.63 \pm 19.77$ & $263.05 \pm 22.19$ \\
\hline Observed species & $280.5 \pm 9.87$ & $247 \pm 15.02$ & $242.37 \pm 17.68$ & $221.25 \pm 24.41$ & $228.25 \pm 24.35$ \\
\hline PD whole tree & $18.22 \pm 0.57$ & $16.71 \pm 0.73$ & $16.66 \pm 0.75$ & $15.18 \pm 1.31$ & $15.58 \pm 1.00$ \\
\hline Shannon & $5.98 \pm 0.19$ & $4.54 \pm 0.33$ & $4.68 \pm 0.49$ & $3.7 \pm 0.47$ & $3.88 \pm 0.53$ \\
\hline Simpson & $0.96 \pm 0.00$ & $0.86 \pm 0.05$ & $0.87 \pm 0.04$ & $0.73 \pm 0.08$ & $0.75 \pm 0.08$ \\
\hline Goods coverage & $0.99 \pm 0.0003$ & $0.99 \pm 0.0002$ & $0.99 \pm 0.0004$ & $0.99 \pm 0.0002$ & $0.99 \pm 0.0003$ \\
\hline Data show as mean $\pm \mathrm{SD}$. N = 8. & & & & \\
\hline
\end{tabular}

In order to clarify the detailed differences of intestinal flora between different groups, we drew scatter diagrams of Top 6 flora content and then compared their differences. The compositions of Firmicutes, Verrucomicrobia and Actinobacteria in 
UC model mice are significantly increased as compared with healthy mice, whereas Bacteroidetes and Proteobacteria are significantly reduced (Fig. 1B). Although there were slight differences in intestinal flora content (Table 2), the trends of microbiome in Mesalazine-treated mice and UC model mice were completely consistent, indicating that control drug Mesalazine didn't show significant modification on gut microbiome community. In contrast, M10 and Myricetin modified the composition of gut microbiota in UC mice. The proportions of Verrucomicrobia and Actinobacteria were significantly increased in the M10-treated mice and Myricetin-treated mice as compared with healthy mice, whereas the proportions of Bacteroidetes and Proteobacteria are significantly reduced. We have noticed that the proportions of Firmicutes showed no difference in the M10-treated mice and the Myricetin-treated mice when compared with healthy mice, whereas there are decreased as compared to UC model mice.

Table 2

The contents of gut microbiome community after drugs treatment at phylum level

\begin{tabular}{|c|c|c|c|c|c|c|}
\hline Group & Firmicutes & Verrucomicrobia & Bacteroidetes & Proteobacteria & Actinobacteria & $\begin{array}{l}\text { Candidatus } \\
\text { Saccharibacteria }\end{array}$ \\
\hline $\begin{array}{l}\text { Healthy } \\
\text { control }\end{array}$ & $\begin{array}{l}34.57 \pm \\
7.19\end{array}$ & $3.87 \pm 5.32$ & $49.35 \pm 6.17$ & $10.67 \pm 3.50$ & $1.05 \pm 0.59$ & $0.40 \pm 0.19$ \\
\hline UC model & $\begin{array}{l}47.41 \pm \\
13.20\end{array}$ & $31.34 \pm 10.67$ & $15.29 \pm 3.29$ & $2.94 \pm 0.62$ & $2.87 \pm 0.87$ & $0.13 \pm 0.07$ \\
\hline Mesalazine & $\begin{array}{l}47.15 \pm \\
15.87\end{array}$ & $23.52 \pm 14.68$ & $24.00 \pm 15.93$ & $2.04 \pm 0.82$ & $3.11 \pm 2.98$ & $0.12 \pm 0.09$ \\
\hline M10 & $\begin{array}{l}31.26 \pm \\
10.09\end{array}$ & $50.10 \pm 8.48$ & $13.63 \pm 5.05$ & $2.29 \pm 1.25$ & $2.50 \pm 2.44$ & $0.19 \pm 0.30$ \\
\hline Myricetin & $\begin{array}{l}33.62 \pm \\
10.33\end{array}$ & $47.19 \pm 9.50$ & $13.67 \pm 3.67$ & $2.41 \pm 0.96$ & $2.91 \pm 1.92$ & $0.19 \pm 0.21$ \\
\hline
\end{tabular}

\section{M10 and Myricetin normalize alpha diversity of gut microbiota in mice}

We further investigated the richness and evenness of gut microbiome among five groups of mice. Alpha diversity (a diversity) revealed a significant change in drug-treatment groups as compared with healthy control mice. All parameters represented alpha diversity in drug-treatment groups were lower than healthy group, indicating that community richness was decreased in all experimental groups. Moreover, M10-treated mice and Myricetin-treated mice demonstrated significant difference as compared to UC model mice (Fig. 2). The detailed results are shown in Table 1.

\section{M10 and Myricetin normalize Beta diversity in intestinal microbiota of UC mice}

To examine the weighted UniFrac distance, all groups were separated on PCoA plot $(P=0.001)$, Adonis plot $(P=0.001)$ and NMDS plot (Fig. 3). It shows that the samples of Mesalazine-treated group have the largest intra-group difference, while other groups have small intra-group difference. At PCoA1 (57.24\%; P< 0.001), healthy mice is significantly different with other four groups of mice. However, at PCoA2 (23.25\%; P = 0.045), chronic UC model mice and Mesalazine-treated mice are different with healthy control mice, while M10-treated mice and Myricetin-treated mice are similar with healthy control mice (Fig. 3A). In Adonis analysis, we found that at PCoA1 (57.24\%), healthy control group is significantly different with other four groups. However, at PCoA2 (23.25\%), M10-treated mice and Myricetin-treated mice are very similar with healthy control mice (Fig. 3B). The results of NMDS analysis (Fig. 3C) are consistent with PCoA and Adonis analysis, indicating that M10 and Myricetin could normalize the gut microenvironment in mice with UC. 
As regards to the analysis of bacteria differential abundance, relative abundance of intestinal flora at genuss level in top 20 differential species was displayed in Fig. 4. We found numerous changes of gut microbiota, as follows: 1) the proportions of Akkermansia, Clostridium XIVa, Olsenella, Clostridium IV, Ruminococcus, Parvibacter and Romboutsia were more abundant in UC model group than that of healthy mice $(\mathrm{P}<0.05)$, and the proportions of Desulfovibrio, Parasutterella, Acetatifactor, Oscillibacter, Barnesiella, Parabacteroides, Flavonifractor, Alistipes, Lactobacillus, Prevotella, Odoribacter and Saccharibacteria_genera_incertae_sedis were significantly decreased $(P<0.05) ; 2)$ The proportions of Akkermansia, Bacteroides, Olsenella, Clostridium IV, Ruminococcus, Parvibacter and Romboutsia were more abundant in Mesalazine-treated group than that of healthy control group $(\mathrm{P}<0.05)$, and the proportions of Desulfovibrio, Parasutterella, Acetatifactor, Oscillibacter, Barnesiella, Parabacteroides, Flavonifractor, Lactobacillus, Odoribacter, Prevotella and Saccharibacteria_genera_incertae_sedis were decreased $(\mathrm{P}<0.05)$; 3 ) The proportions of Akkermansia and Ruminococcus were more abundant in M10-treated group than that of healthy control group $(\mathrm{P}<0.05)$, and the proportions of Desulfovibrio, Parasutterella, Acetatifactor, Oscillibacter, Barnesiella, Parabacteroides, Flavonifractor, Alistipes, Lactobacillus, Prevotella, Odoribacter and Saccharibacteria_genera_incertae_sedis were decreased $(P<0.05) ; 4)$ The proportions of Akkermansia and Ruminococcus were more abundant in the Myricetin-treated mice than that of healthy control mice $(\mathrm{P}<0.05)$, and the proportions of Desulfovibrio, Parasutterella, Acetatifactor, Oscillibacter, Barnesiella, Parabacteroides, Flavonifractor, Alistipes, Lactobacillus, Prevotella, Odoribacter and Saccharibacteria_genera_incertae_sedis were significantly decreased $(P<0.05)$. As comparing to UC model mice, we found some changes as follows: 1) The proportions of Oscillibacter, Ruminococcus, Clostridium IV, Desulfovibrio, Parabacteroides, Parvibacter and Olsenella were significantly decreased in M10-treated group $(\mathrm{P}<0.05) ; 2)$ The proportions of Desulfovibrio, Parabacteroides, Clostridium IV, Parasutterella, Ruminococcus, Parasutterella and Romboutsia were significantly decreased in Myricetin-treated group $(\mathrm{P}<0.05)$. All above results confirm that after treatment with M10 and Myricetin, the composition of intestinal flora of UC mice is closer to that of normal mice. In addition, the results of heatmap for all horizontally differentiated species are consistent with this conclusion (Supplementary Fig. 1).

\section{Identification of signature bacteria as mediators to accelerate the reshape of inflammed colon}

In the UC mice, oral M10 and Myricetin reshaped gut microbiota. To confirm both statistical and biological taxonomic differences between gut microbiota and define biomarker among five groups mice, the LEfSe algorithm with a logarithmic LDA score cutoff $\geq 2.0$ (LDA $\geq 2, P<0.05$ ) was performed, and a total of 110 biomarkers were found. In order to identify the signature bacteria as mediators which accelerate the repairing of inflammed colon, we adopted stricter screening criteria (LDA $\geq 4$ ) than others. As expected in healthy control mice, species such as Bacteroidetes, Proteobacteria, Erysipelotrichaceae_incertae_sedis, Porphyromonadaceae, Enterobacteriaceae, Deltaproteobacteria, Escherichia_Shigella, Desulfovibrionales, Anaerotruncus, Betaproteobacteria, Sutterellaceae, Parasutterella, Acetatifactor, Streptococcus, Streptococcaceae and Barnesiella exhibited elevated proportions, whereas in M10-treated mice, the abundance of Akkermansia, Verrucomicrobiae, Gemmiger and Clostridium XIVa are significantly increased. Mesalazine-treated mice demonstrated increased abundance of Ruminococcaceae. However, UC mice are characterized by increased abundance of Enterobacter, Clostridium, Ruminococcus and Parvibacte (Fig. 5).

\section{M10 and Myricetin changed the metabolic function of gut microbiota}

The PICRUSt algorithm (phylogenetic investigation of communities by reconstruction of unobserved states) was used to evaluate functional differences of bacteria in different groups. The differential pathways analysis was performed against KEGG, and analyzed and plotted (Fig. 6). By this way we can estimate the degree of influence of each component's metabolic pathway (KEGG pathway) on different effects, and find metabolic pathway that has a significant difference in sample division (the default screening condition is LDA $>2$, The figure takes on third level). The analysis indicated that DSS-induced disruption in colon epithelium was reverted by M10 and Myricetin, which demonstrated similar histopathologic features to healthy control mice. The alteration of abundance of gut microbiota in M10-treated mice was 
strongly increased both in biosynthesis and degradation activities to improve gut microenvironment, such as sulfur metabolism, pyruvate metabolism, steroid biosynthesis and unsaturated fatty acid biosynthesis. Moreover, Myricetintreated mice demonstrated significant increased of Ascorbate and aldarate metabolism and lipid metabolism.

\section{Discussion}

In current study, we aim to determine if the effects of Myricetin and its derivative M10 on UC are associated to their activity of modification the richness and diversity of gut microbiota. Previously, M10 demonstrated higher activities of antiulcerative colitis than Myricetin. M10 prevented DSS-induced UC by inhibiting colonic mucosal cell necrosis and remodeling intestinal barrier [21]. M10 possessed higher activity than Myricetin in prevention of UC. Both M10 and Myricetin did not absorb into systemic circuit but played local activities in intestinal tract [20]. We thus speculate that the effects of M10 on UC are associated to its biological role in the modification of gut microbiota. Our result demonstrated that M10 and Myricetin affect the gut microenvironment (a- or $\beta$-diversity) as compared with UC model mice (Fig. 2 and Fig. 3). We have noticed that although the abundance of gut microbiota is lower in M10-treated mice and Myricetin-treated mice as compared with UC mice, the diversity of these mice are more similar with healthy control mice, which means M10 and Myricetin could reshape gut microenvironment in UC mice model (Supplementary Fig. 1). Current results are consistent with a previous study that chronic UC gut microbiomes exhibit general decreases in taxonomic diversity relative to healthy gut microbiomes [30]. The differentially affected bacteria generally fell into two major categories, Firmicutes and Actinobacteria. The proportions of Firmicutes and Actinobacteria in UC mice are significantly increased as compared to healthy control group, while M10 and Myricetin treatment resulted in normalizing the proportion of Firmicutes and Actinobacteria in gut microbiota.

At genuss level, we noted that the protective effect of M10 and Myricetin on DSS-induced ulcerative colitis was associated to the increase of probiotics, such as Akkermansia, and the inhibition of pathogens, such as Ruminococcus and Parabacteroides. Ruminococcus contribute to inflammation through production of inflammatory polysaccharide and are associated with thinning of intestinal mucosa and impairment of intestinal barrier function [31-33]. Paraprevotella is a pathogenic bacterium. Indeed, according to previous studies, its abundance is correlated with the incidence of UC and proinflammatory cytokine secretion by colonic epithelial cells [34]. A previous study showed that stachyose could change gut microbiota composition with a higher level of Akkermansia [35]. Akkermansia can protect from liver injury, reinforce gut barrier function, and reduce inflammation [36]. Correlation coefficient analysis demonstrated that Akkermansia abundance was negative correlated with Paraprevotella (Supplementary Fig. 2). Hence, M10 could deplete the proportion of Ruminococcus and Parabacteroides, resulted to the proportion of Akkermansia, thus leading to alleviating chronic UC. Furthermore, M10 reshaped intestinal barrier, which prevented intestinal flora invasion into blood in UC mice [21].

Overall, UC mice demonstrated the increases of Firmicutes and Actinobacteria as compared to healthy control mice. Oral M10 and Myricetin normalized the proportion of Firmicutes and Actinobacteria in gut microbiota. M10 also increased both biosynthesis and degradation activities, resulting to improving the metabolism of sulfur, pyruvate, steroid biosynthesis and unsaturated fatty acid biosynthesis in gut microenvironment. Myricetin and M10 could prevent UC through modifying the composition of gut microbiota. Combined with pharmacologic effects of Myricetin and M10 in these UC mice, we conclued that the effects of Myricetin and M10 on UC were associated to the modification of intestinal microbiota in the environment of chronic ulcerative colitis.

\section{Declarations}

Ethics approval and consent to participate: This project related to animal protocol was approved by Capital Medical University Institutional Animal Care and Use Committee (permit no. AEEI-2018-043). All participates consented the results and current form of the manuscript. 
Consent for publication: All participates agreed to transfer the right of publication to Gut Pathogens

Data availability statement: The datasets used and/or analyzed during the current study are available from the corresponding author on reasonable request.

Competing interests: The authors have no conflicts of interest.

Funding information: This work was supported by National Natural Science Foundation of China (91629303/81673449/81872884/81973350) and Beijing Natural Science Foundation and Scientific Research Program of Municipal Commission of Education (KZ201710025020/KZ201810025033).

Authors' contributions: Qu XJ and Cui SX received and designed the project. Miao RR, Zhan S and Hu XT performed all the experiments. Miao RR performed statistical analysis.

Acknowledgements: We thanked professor Yu for her valuable comments in designing the experiments.

Supporting information: Additional supporting information can found online in Supporting Information section.

\section{References}

1. Baumgart DC, Carding SR. Inflammatory bowel disease: cause and immunobiology. Lancet. 2007;369:1627-40.

2. $\mathrm{Ng} \mathrm{SC}$, Shi HY, Hamidi N, et al. Worldwide incidence and prevalence of inflammatory bowel disease in the $21 \mathrm{st}$ century: a systematic review of population-based studies. Lancet. 2017;390:2769-78.

3. Ungaro R, Mehandru S, Allen PB, et al. Ulcerative colitis Lancet. 2017;389:1756-70.

4. Cleynen I, Vermeire $S$. The genetic architecture of inflammatory bowel disease: past, present and future. Curr. Opin Gastroenterol. 2015;31:456-63.

5. McGovern DP, Kugathasan S, Cho JH. Genetics of inflammatory bowel diseases. Gastroenterology. 2015;149:116376.

6. Rogler G, Vavricka S. Exposome in IBD: recent insights in environmental factors that influence the onset and course of IBD. Inflamm Bowel Dis. 2015;21:400-8.

7. Kostic AD, Xavier RJ, Gevers D. The microbiome in inflammatory bowel disease: current status and the future ahead. Gastroenterology. 2014;146:1489-99.

8. Manichanh C, Borruel N, Casellas F, et al. The gut microbiota in IBD. Nat. Rev Gastroenterol Hepatol. 2012;9:599-608.

9. Goodrich JK, Waters JL, Poole AC, et al. Human genetics shape the gut microbiome. Cell. 2014;159:789-99.

10. Machiels K, Joossens M, Sabino J, et al. A decrease of the butyrate-producing species Roseburia hominis and Faecalibacterium prausnitzii defines dysbiosis in patients with ulcerative colitis. Gut. 2013;63:304833.

11. Semwal DK, Semwal RB, Combrinck S, et al. Myricetin: a dietary molecule with diverse biological activities. Nutrients. 2016;8:90.

12. Yang C, Lim W, Bazer FW, et al. Myricetin suppresses invasion and promotes cell death in human placental choriocarcinoma cells through induction of oxidative stress. Cancer Lett. 2017:399.

13. Imhann F, Vich Vila A, Bonder MJ, et al. Interplay of host genetics and gut microbiota underlying the onset and clinical presentation of inflammatory bowel disease. Gut. 2018;67:108-19.

14. Zhernakova A, Kurilshikov A, Bonder MJ, et al. Population-based metagenomics analysis reveals markers for gut microbiome composition and diversity. Science. 2016;352:565-9. 
15. Chu H, Khosravi A, Kusumawardhani IP, et al. Gene-microbiota interactions contribute to the pathogenesis of inflammatory bowel disease. Science. 2016;352:1116-20.

16. Li M, Chen J, Yu X, et al. Myricetin suppresses the propagation of hepatocellular carcinoma via down-regulating expression of YAP. Cells 2019;8:358 - 74.

17. da Silva-Maia JK, Batista AG, Correa LC, et al. Aqueous extract of berry (Plinia jaboticaba) byproduct modulates gut microbiota and maintains the balance on antioxidant defense system in rats. J. Food. Biochem, 2019;43.

18. Yao Y, Xie Y, Hong C, et al. Development of a Myricetin/hydroxypropyl- $\beta$-cyclodextrin inclusion complex: preparation, characterization, and evaluation. Carbohyd Polym. 2014;110:329.

19. Zhu S, Yang C, Zhang L, et al. Development of M10, myricetin-3-O- $\beta$-d-lactose sodium salt, a derivative of myricetin as a potent agent of anti-chronic colonic inflammation. Eur J Med Chem. 2019;174:9-15.

20. Wang F, Song ZY, Qu XJ, et al. M10, a novel derivative of Myricetin, prevents ulcerative colitis and colorectal tumor through attenuating robust endoplasmic reticulum stress. Carcinogenesis. 2018;39:889.

21. Zhou XL, Yang J, Qu XJ, et al. M10, a Myricetin-3-O-b-D-lactose sodium salt, prevents ulcerative colitis through inhibiting necroptosis in mice. Front Pharmacol. doi.org/10.3389/fphar.2020.557312.

22. Sartor RB, Wu GD. Roles for intestinal bacteria, viruses, and fungi in pathogenesis of inflammatory bowel diseases and therapeutic approaches. Gastroenterology. 2017;152:327-39.

23. Siniagina M, Markelova M, Laikov A, et al. Cultivated Escherichia coli diversity in intestinal microbiota of Crohn's disease patients and healthy individuals: whole genome data. Data Brief. 2020;28:104948.

24. Pan G, Liu B, Li S, et al Kuijieling. A Chinese medicine alleviates DSS-induced colitis in C57BL/6 J mouse by improving the diversity and function of gut microbiota. FEMS. Microbiol Lett. 2020;367:fnaa082.

25. Meidenbauer JJ, Ta N, Seyfried TN. Influence of a ketogenic diet, fish-oil, and calorie restriction on plasma metabolites and lipids in C57BL/6J mice. Nutr Metab. 2014;11:23.

26. Wang Y, Qian PY. Conservative fragments in bacterial 16S rRNA genes and primer design for 16S ribosomal DNA amplicons in metagenomic studies. PLoS One. 2009;4:e7401-9.

27. Masella AP, Bartram AK, Truszkowski JM, et al. PANDAseq: paired-end assembler for illumina sequences. BMC Bioinformatics. 2012;13:31.

28. Edgar RC. UPARSE: Highly accurate OTU sequences from microbial amplicon reads. Nat Methods. 2013;10:996-8.

29. Paul FK, Josephine YA. Bacterial diversity in aquatic and other environments: what 16S rDNA libraries can tell us. FEMS. Microbiol Eco. 2004;47:161-77.

30. Lundberg DS, Yourstone S, Mieczkowski P, et al. Practical innovations for high-throughput amplicon sequencing. Nat methods. 2013;10:999-1002.

31. Huttenhower C, Kostic AD, Xavier RJ. Inflammatory bowel disease as a model for translating the microbiome. Immunity. 2014;40:843-54.

32. Lloyd Price J, Arze C, Ananthakrishnan AN, et al. Multi-omics of the gut microbial ecosystem in inflammatory bowel diseases. Nature. 2019;569:655-62.

33. Henke MT, Kenny DJ, Cassilly CD, et al. Ruminococcus gnavus, a member of the human gut microbiome associated with Crohn's disease, produces an inflammatory polysaccharide. Proc. Natl. Acad. Sci. 2019;116:12672-7.

34. Feng Q, Liang S, Jia H, et al. Gut microbiome development along the colorectal adenoma-carcinoma sequence. Nat Commun. 2015;6:6528.

35. He L, Zhang F, Jian Z, et al. Stachyose modulates gut microbiota and alleviates dextran sulfate sodium-induced acute colitis in mice. Saudi J Gastroenterol. 2020;26:153-9.

36. Wu W, Lv L, Shi D, et al. Protective effect of Akkermansia muciniphila against immune-mediated liver injury in a mouse model. Front. Microbiol. 2017;8:1804. 
Fig. 1
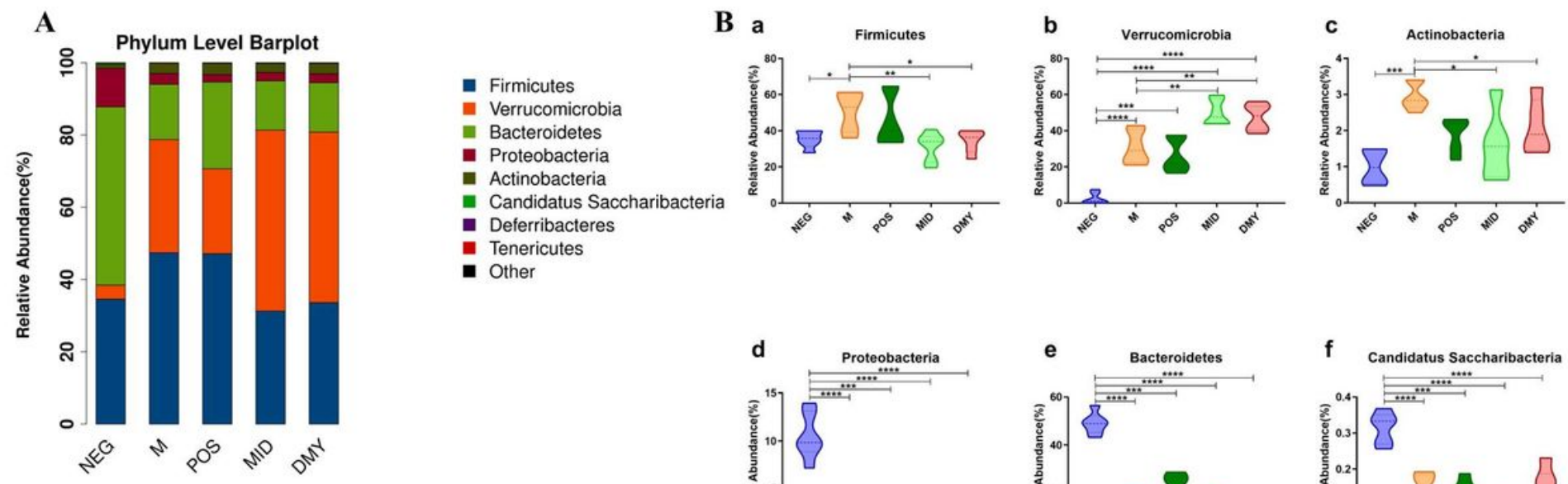

Verrucom

- Bacteroidetes

- Proteobacteria

- Candidatus Saccharibacteri

- Deferribacteres

- Tenericutes

- Other
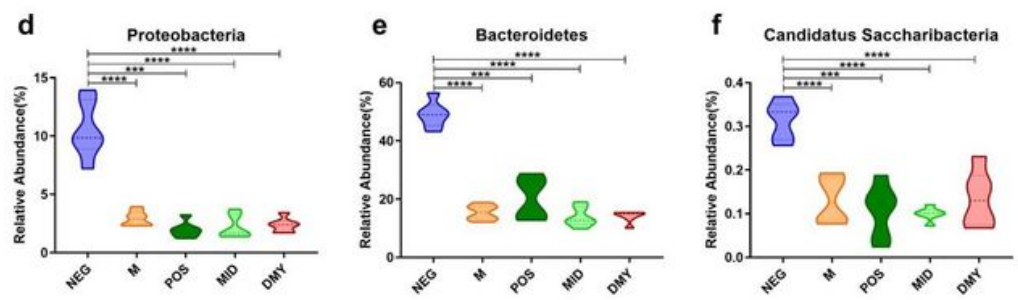

Figure 1

M10 and Myricetin reshaped gut microbiome taxonomic diversity in UC mice. (A) At phyla level, gut microbiota composition was different among healthy mice, UC model mice, Mesalazine-treated mice, M10-treated and Myricetintreated mice. Horizontal axis is the name of group, and vertical axis is relative abundance of species. Different colors correspond to different species, and the length of color block indicates relative abundance of species represented by color. (B) Specific differences in the richness of five groups at phylum levels of Firmicutes, Verrucomicrobia, Bacteroidetes, Proteobacteria, Actinobacteria, and Candidatus Saccharibacteria. ${ }^{*} P<0.05 ;{ }^{*} P<0.01 ; * \star * P<0.001 ; * \star * * P<0.0001 . N=8$. Abbreviations: NEG group: healthy control group; M group: UC model group; POS group: Mesalazine-treated group; MID group: M10-treated group; DMY group: Myricetin-treated group.

Fig. 2
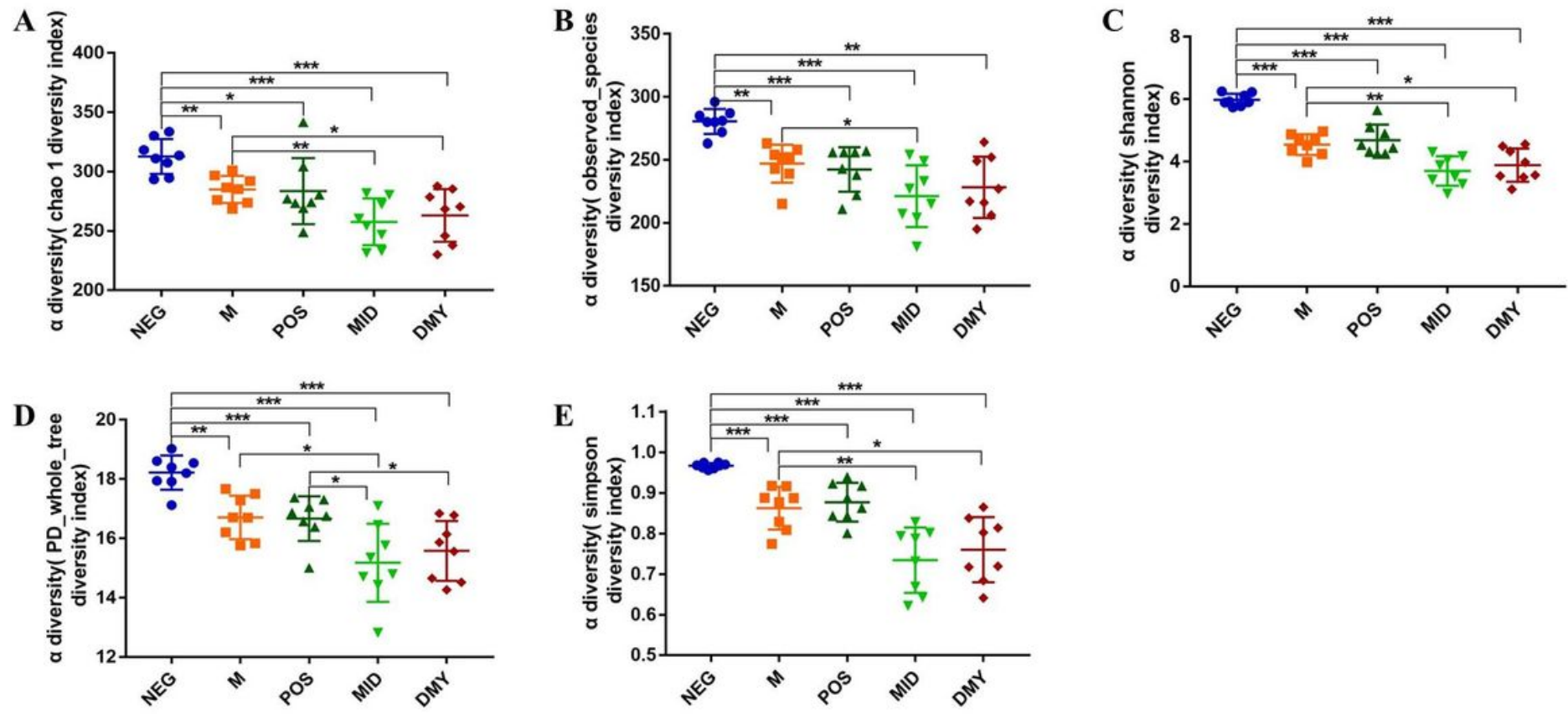


\section{Figure 2}

M10 and Myricetin normalized alpha diversity in intestinal microbiota of UC mice. Alpha diversity was used to analyze a single species in a sample, including (A) chao 1 index; (B) observed_species index; (C) PD_whole_tree index; (D) Shannon index; (E) Simpson index. Rank test was used to analyze the difference among five groups. ${ }^{\star} P<0.05$; ${ }^{\star \star} P<0.01$; ${ }^{\star \star \star} P<$ 0.001. $N=8$. Abbreviations: NEG group: Healthy control group; M group: UC model group; POS group: Mesalazine-treated group; MID group: M10-treated group; DMY group: Myricetin-treated group.

Fig. 3
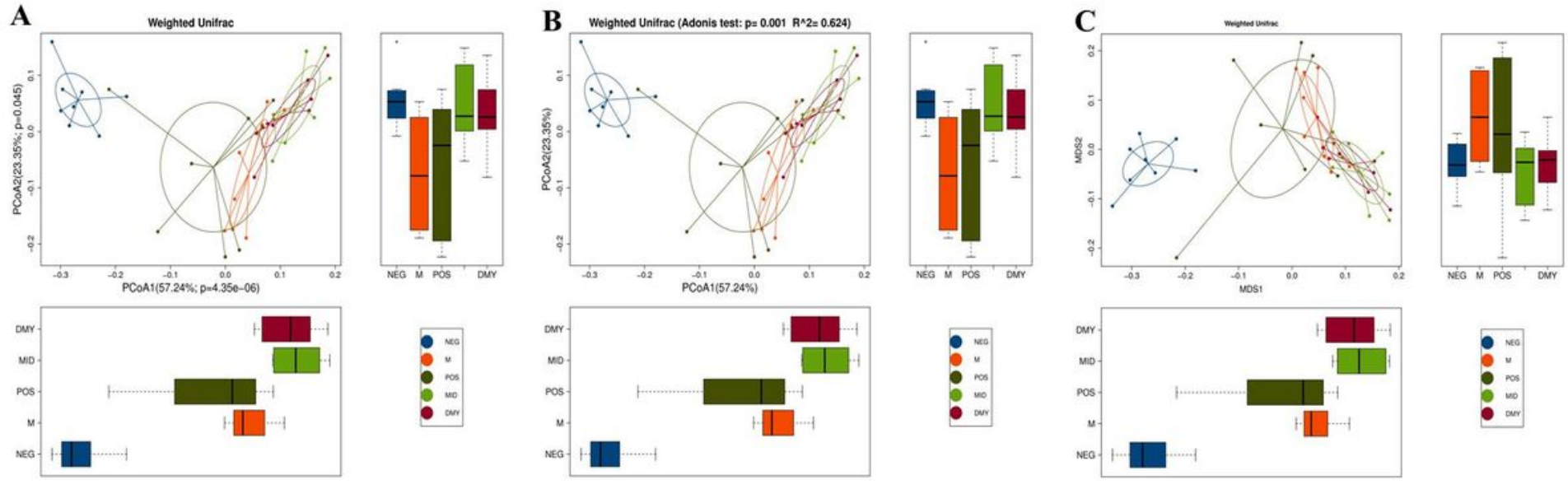

PCoA

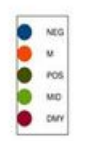

Adonis
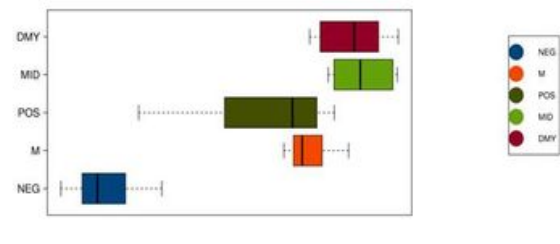

NMDS

Figure 3

M10 and Myricetin normalized Beta diversity in intestinal microbiota of UC mice. (A) Principal Coordinates Analysis (PCoA) ordination plot on weighted UniFrac distance matrix. (B) Adonis analysis of PCoA diagram on weighted UniFrac distance matrix. (C) NMDS analysis on weighted UniFrac distance matrix. Each subject is identified by a point. Healthy control group are represented in blue, chronic UC model group are represented in orange, Mesalazine-treated group are represented in Olive green, M10-treated group are represented in green, Myricetin-treated group are represented in dark red. Abbreviations: NEG group: Healthy control group; M group: UC model group; POS group: Mesalazine-treated group; MID group: M10-treated group; DMY group: Myricetin-treated group. 
Fig. 4

A

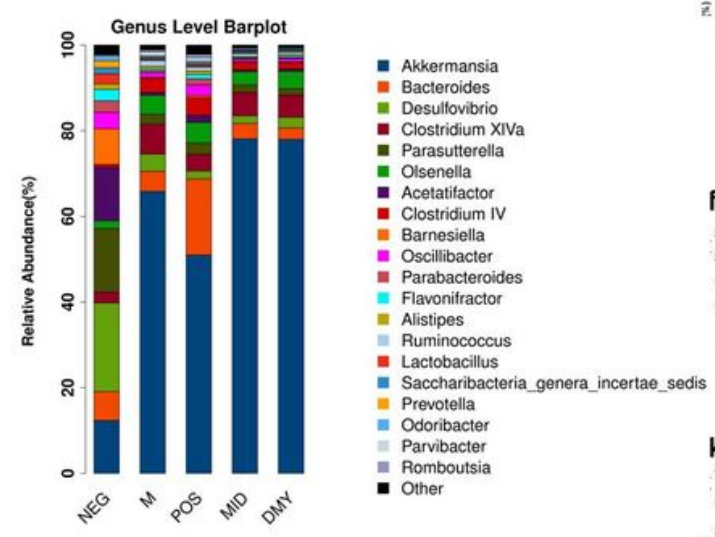

B
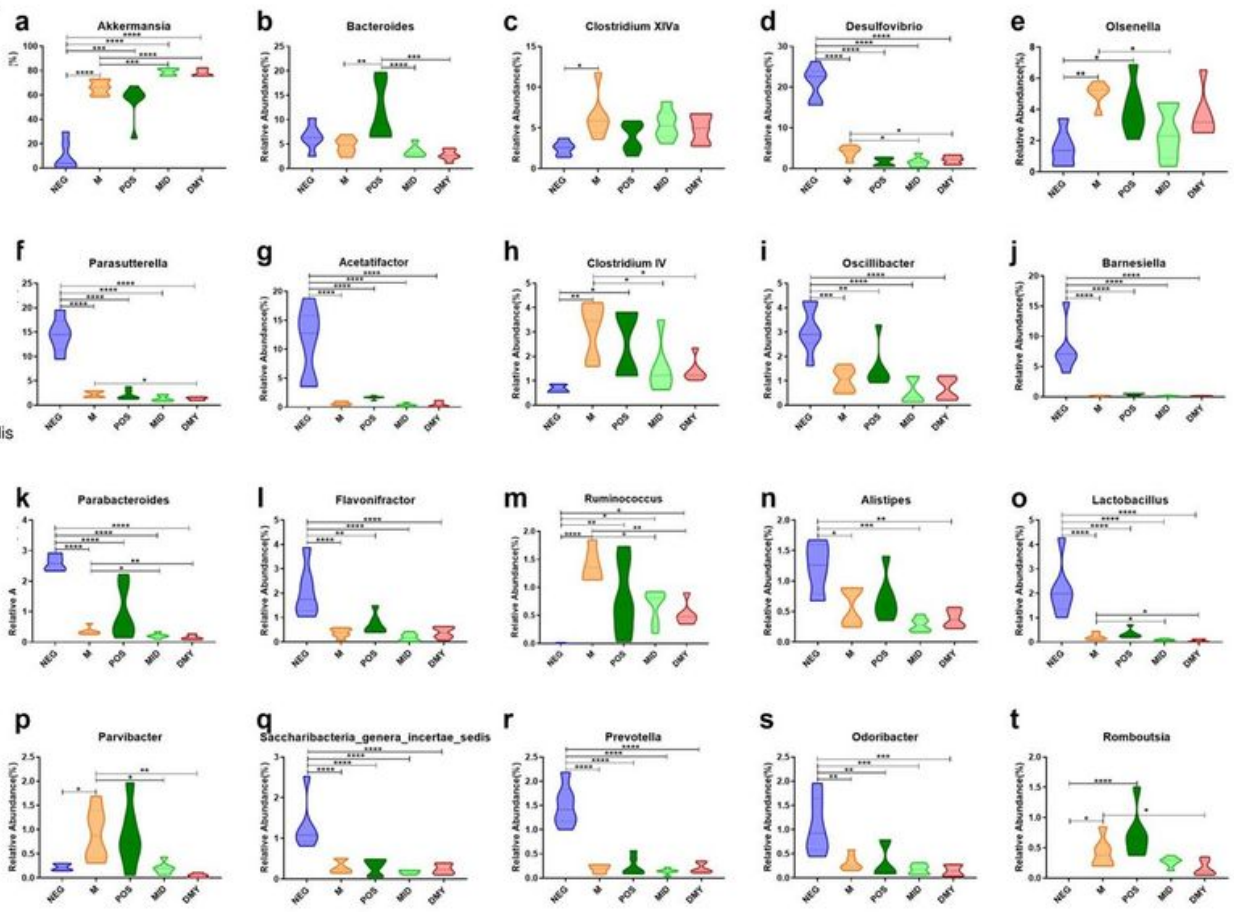

Figure 4

M10 and Myricetin change the proportion of probiotics and pathogens to relieve UC symptoms. (A) At genuss level, gut microbiota composition were different among healthy control group, UC model group, Mesalazine-treated group, M10treated group and Myricetin-treated group. Horizontal axis is the name of samples, and vertical axis is relative abundance of species. Different colors correspond to different species, and length of color block indicates relative abundance of species represented by color. (B) Detailed gut microbiota richness in different bacteria (a-t). ${ }^{*} \mathrm{P}<0.05 ;{ }^{*} \mathrm{P}<0.01$; ${ }^{\star \star \star} \mathrm{P}<$ $0.001 ;{ }^{* \star * *} \mathrm{P}<0.0001 . \mathrm{N}=8$. Abbreviations: NEG group: healthy control mice; $\mathrm{M}$ group: UC model mice; POS group: Mesalazine-treated mice; MID group: M10-treated mice; DMY group: Myricetin-treated mice. 
Fig. 5

A

Cladogram

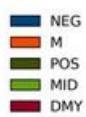

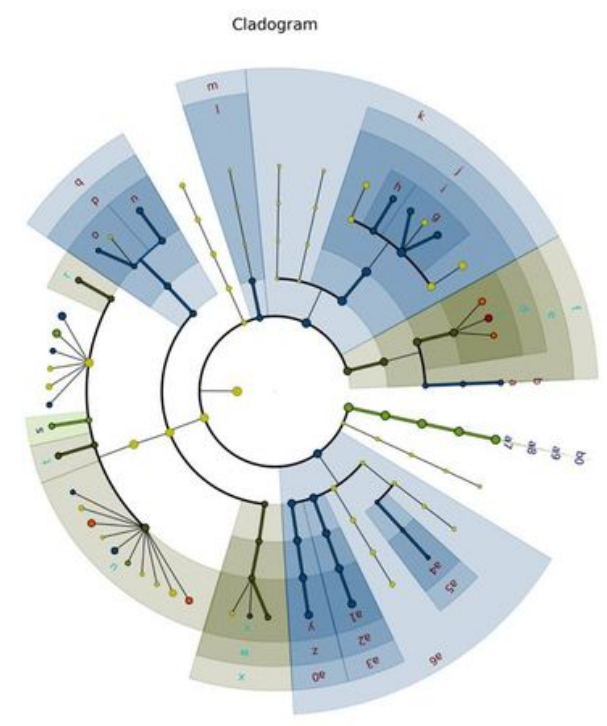

B

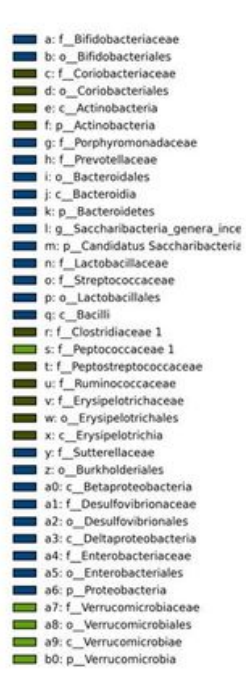

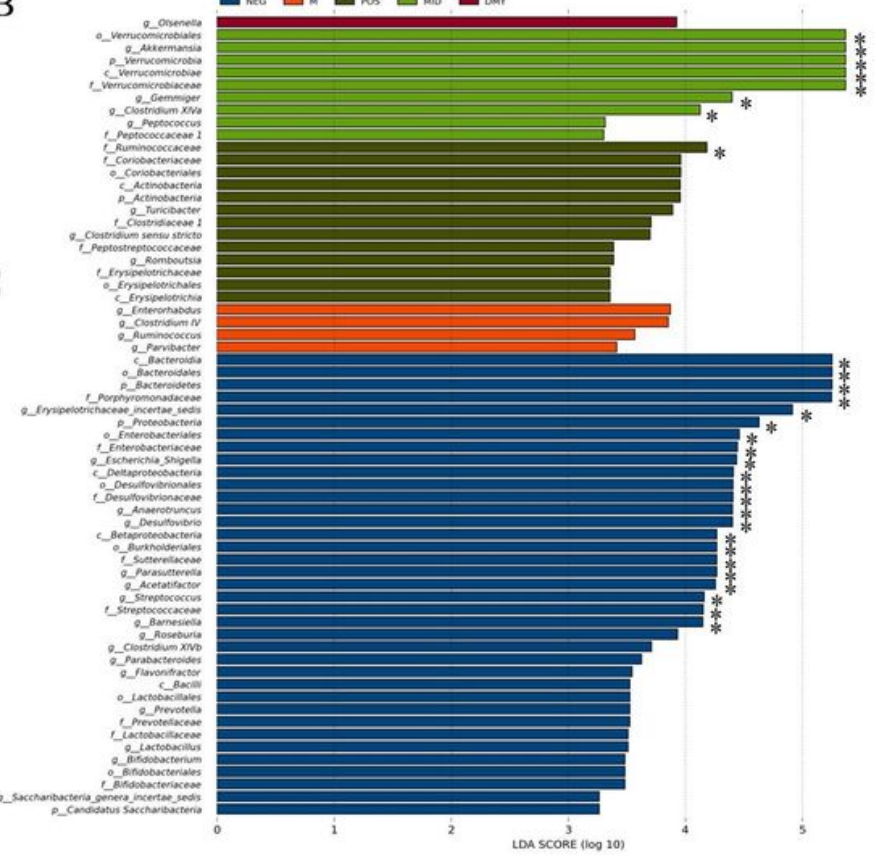

Figure 5

Identification of signature bacteria as mediators to accelerate the ability of repairing superficial mucosal inflammation in colon. (A) Cladogram displays the taxonomic tree of differentially abundant taxa. (B) Histogram represents the linear discriminant analysis (LDA) scores of bacteria with significant differential abundance between compared groups, identified by different colors. *features with LDA score > 4. Abbreviations: NEG group: healthy control mice; M group: chronic UC model; POS group: Mesalazine-treated mice; MID group: M10-treated mice; DMY group: Myricetin-treated mice. 


\section{Fig. 6}

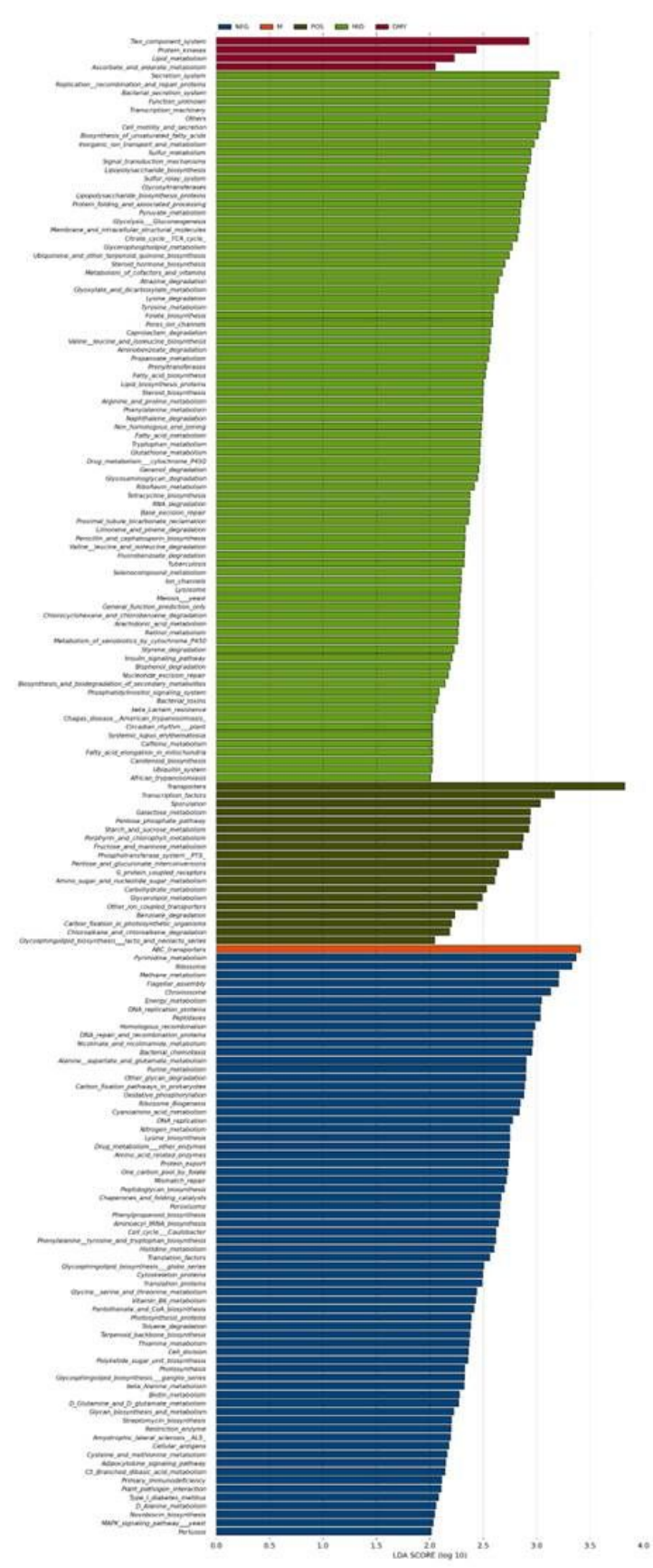

Figure 6

M10 and Myricetin changed the metabolic function of gut microbiota. This diagram shows LEfSe analysis of statistical KEGG difference pathway. Abscissa is log value obtained by LDA (linear regression analysis) of KEGG pathway that has a significant role in different groups. The symmetry shows that LDA score is an absolute value. The ordinate represents different KEGG signal pathways. Abbreviations: NEG group: Healthy control group; M group: UC model group; POS group: Mesalazine-treated group; MID group: M10-treated group; DMY group: Myricetin-treated group.

\section{Supplementary Files}

This is a list of supplementary files associated with this preprint. Click to download.

- Supplementarydata.doc 